TecnoLógicas

ISSN-p 0123-7799

ISSN-e 2256-5337

Vol. 23, No. 49 , pp. $72-80$

Sep-dic de 2020

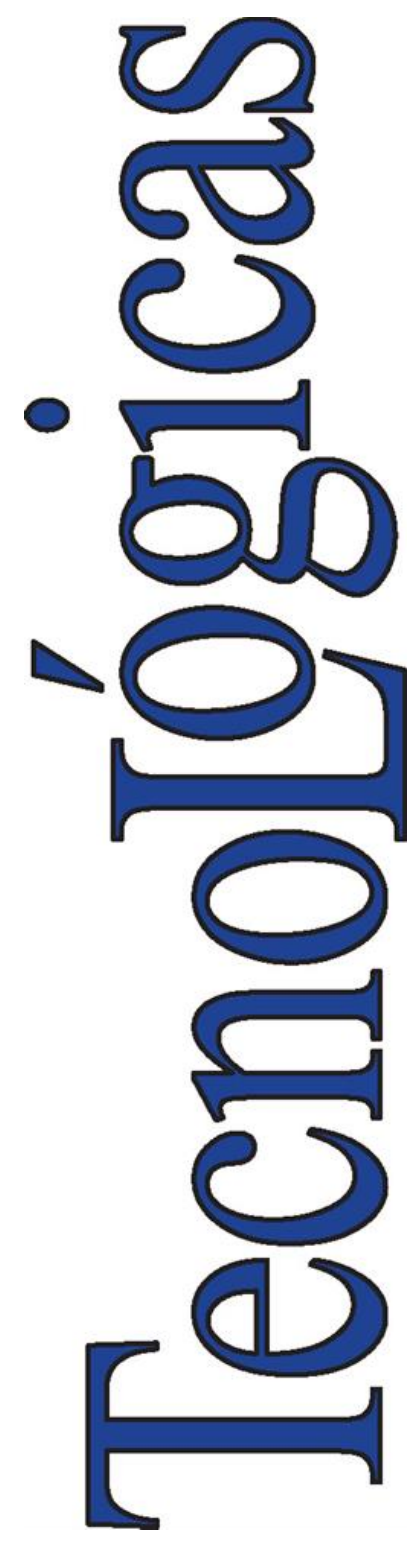

(C) Instituto Tecnológico Metropolitano Este trabajo está licenciado bajo una Licencia Internacional Creative Commons Atribución (CC BY-NC-SA)

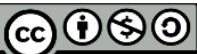

Artículo de investigación/Research Article

\section{Metodología para la selección de la mejor variante entre electrodos de doble revestimiento para recargue}

\section{Methodology for the Selection of the Best Variant Between Double Coated Electrodes for Hardfacing}

Odonel González Cabrera (D) 1 ,

Carlos Rene Gómez Pérez ${ }^{\text {D }}{ }^{2}$, y

Uvedel Bernabé Del Pino Paz (D) 3

Recibido: 3 de marzo de 2020

Aceptado: 26 de mayo de 2020

Cómo citar / How to cite

O. González-Cabrera, C. R. Gómez-Pérez, U. B. Del Pino-Paz, "Metodología para la selección de la mejor variante entre electrodos de doble revestimiento para recargue", TecnoLógicas, vol. 23, no. 49, pp. 72-80, 2020. https://doi.org/10.22430/22565337.1608
1 PhD. en Ciencias Técnicas, Centro de Información y Gestión Tecnológica, Santa Clara-Cuba, ogonzalez@ciget.vcl.cu

2 PhD. en Ciencias Técnicas, Centro de Investigaciones de Soldadura, Universidad Central "Marta Abreu" de Las Villas, Santa ClaraCuba, crene@uclv.edu.cu

3 PhD. en Ciencias Físico Matemáticas, Facultad de Matemática, Física y Computación, Universidad Central "Marta Abreu" de Las Villas, Santa Clara-Cuba, uvedel@uclv.edu.cu 


\title{
Resumen
}

El presente trabajo persigue establecer una metodología que permita la selección de la mejor variante a emplear ante determinadas condiciones de un consumible para enfrentar fenómenos de desgaste, y que esta conjugue los principales parámetros que puedan arrojar criterio para ello. Entre los criterios seleccionados como parte de la metodología, se encuentran los ensayos de estabilidad del arco y los relacionados con la determinación de los parámetros de consumo de los electrodos, los cuales permiten la evaluación del comportamiento operativo. Para el primero, se calculan los valores de la conductividad del arco, y para el segundo, el coeficiente de deposición. En cuanto a la evolución del depósito, se decide por la medición de la resistencia al desgaste por medio de la pérdida de masa, empleando el ensayo de abrasión con espiga. Los valores obtenidos en estas tres pruebas se aleatorizan y se someten a criterio de expertos. Las valoraciones de los expertos se procesan y se obtiene, matemáticamente, la ponderación que los expertos otorgan a cada parámetro expresado, a través de ecuaciones de regresión. Se selecciona unánimemente como la mejor variante de electrodo doblemente recubierto, la que posee la carga de aleación externa. Los resultados del análisis realizado para la selección de la mejor variante sugieren que la resistencia al desgaste, evaluada a través de la pérdida de masa, es el criterio de mayor relevancia para la selección de electrodos destinados al recargue de piezas.

\section{Palabras clave}

Electrodos para recargue, criterio de expertos, mejor variante, resistencia al desgaste.

\begin{abstract}
This study aims to establish a methodology for selecting the best variant to be employed under certain conditions of a consumable electrode to address wear phenomena. In addition, it should combine the main parameters that can provide criteria for this purpose. As part of the methodology, some of the selected criteria include arc stability tests and tests to determine the consumption parameters of electrodes which assess their operational behavior. For the former, arc conductivity is calculated; and for the later, the deposition coefficient. Regarding the evolution of the deposit, we decide to measure wear resistance based on loss of mass using the pin abrasion test. The values obtained in these three tests are randomized and subjected to expert criteria. Then, the experts' assessments are processed, and the weight they give to each parameter is estimated mathematically through regression equations. The variant with the external alloying charge is unanimously selected as the best double-coated electrode variant. The results of the analysis performed to choose the best variant suggest that wear resistance (assessed based on loss of mass) is the most relevant criterion for selecting electrodes intended for hardfacing parts.
\end{abstract}

\section{Keywords}

Hardfacing electrodes, expert criteria, best variant, wear resistance. 


\section{INTRODUCCIÓN}

Es conocida la evolución del uso de los procesos de soldadura por arco eléctrico durante los últimos años [1]. En los países de Latinoamérica, esta tendencia ha sido menos marcada, empleándose hasta el $40 \%$ del metal depositado en países como Argentina. En China y la India, acompañando el notable crecimiento de la producción de acero, se ha observado un marcado aumento del uso de electrodos revestidos. Todo parece indicar que el uso de electrodos revestidos se estabilizará en alrededor del $30 \%$ del metal de soldadura depositado a nivel mundial [2].

El desgaste es parte de los procesos productivos, ya que los metales sufren los efectos de las partículas abrasivas que provocan pérdidas sensibles en los mismos.

La acción de estas partículas en el mecanismo de desgaste depende de múltiples factores, además de las características del material desgastado. La investigación sobre nuevos materiales para recargue es uno de los campos de mayores potencialidades para enfrentar fenómenos como el desgaste de piezas y componentes [3], [4].

El estudio adecuado de los mecanismos de desgaste contribuye en gran porcentaje a realizar la selección del consumible apropiado. En algunos casos, la pieza puede estar sometida a varios factores. No existe el consumible que resista todas las condiciones anteriores, pero, en la práctica, siempre puede encontrarse una solución para cada caso [5].

Varios son los criterios empleados para seleccionar un consumible a emplear en determinada aplicación. Nunca debe realizarse tomando como criterio un solo parámetro. Entre los parámetros empleados se encuentran los parámetros de fusión y deposición, los que permiten estimar la productividad del electrodo y poder compararla con la de otra conocida o ensayada bajo las mismas circunstancias.
Para ello, puede usarse la norma ISO 2401-72 [6], en la que se valoran como principales parámetros de consumo la eficiencia de deposición (RD) y el coeficiente de deposición (D) [7].

La evaluación de la estabilidad del proceso de soldadura empleando la digitalización de las señales experimentales de voltaje y corriente de soldadura [8]-[12]-[13] señala que sus espectros de frecuencia están determinados por la fenomenología del arco eléctrico, en función de las propiedades químico-físicas de las sustancias componentes del plasma y su inercia, entre otros aspectos, lo cual está de acuerdo a lo planteado por [14].

En la soldadura con corriente alterna (CA) suele suceder que las extinciones naturales del arco no siempre terminan con su reencendido en el siguiente semiperíodo del voltaje. Como resultado, la soldadura con CA es la más inestable de todos los procesos de soldadura, mientras la frecuencia de las extinciones de arco es, sin duda, el principal indicador objetivo de su ignición [9]. La estabilidad del arco en soldadura con corriente alterna se evalúa con base en la variación de la tasa media de incremento de la conductividad del espacio de arco $(\mathrm{B}+)$.

Para establecer criterios sobre la resistencia al desgaste a alta tensión se ha empleado el ensayo tipo abrasión con espiga para determinar los valores de este y el coeficiente de fricción de materiales durante deslizamiento [15], [16]. Este ensayo consiste en una prueba comparativa, comúnmente empleada para la evaluación del desgaste abrasivo en depósitos de soldadura [15], a través de la comparación entre la masa de metal perdida durante el ensayo [17].

Como objetivo del presente trabajo se plantea establecer una metodología para la selección de la mejor variante de electrodo a emplear ante el desgaste abrasivo a través de la identificación y uso de los principales parámetros que puedan arrojar criterio para ello. 
No se han encontrado valoraciones sobre estos aspectos en electrodos con una estructura de doble revestimiento aplicados en el recargue, explícitamente en la literatura consultada.

\section{METOdOLOGíA}

\subsection{Materiales}

Para la obtención de los revestimientos en los electrodos estudiados, se precisó preparar dos cargas de materias primas, una para cada uno. Un revestimiento para garantizar los parámetros operativos del electrodo, constituido por un $70 \%$ de la masa seca total, correspondiente a la composición comercial, reconocida internacionalmente como revestimiento rutílico E6012.

Otro revestimiento $(30 \%$ de la masa seca total) se diseñó para aportar la carga de aleación. Las proporciones de 70 y $30 \%$ se asumen según recomendaciones de la literatura [18], y buscando asegurar que la estabilidad del arco no se comprometa con la variación de la posición de la carga de aleación en la estructura del revestimiento.

La carga de aleación está constituida por un $24 \%$ de ferroaleaciones y un $6 \%$ de grafito.

Se fabricaron tres tipos de electrodos con la misma composición química para el núcleo metálico y la carga de aleación ver Tabla 1. Igualmente, se empleó el mismo revestimiento ver Tabla 2 , similar al de un electrodo E6012 [2]. El revestimiento de la carga de aleación para cada tipo de electrodo ocupó la misma área de la sección transversal del revestimiento, por tanto, la misma medida en el volumen y masa totales del revestimiento. El aglutinante empleado también fue común para todos los electrodos: silicato de sodio, con una densidad de 1,44 a $1,48 \mathrm{~g} / \mathrm{cm}^{3}$, ocupando entre un $28 \%$ y $30 \%$ de la masa total del revestimiento.

Las masas de revestimiento y carga de aleación involucradas en cada variante de electrodo se garantizaron iguales, a partir de la consideración del producto de la masa y la densidad de cada componente a través de sus respectivas áreas $\left(\mathrm{A}_{1} \mathrm{y} \mathrm{A}_{2}\right)$, para cada caso (Fig. 1).

Tabla 1. Composición química de las materias primas empleadas en la confección de los electrodos Fuente: elaboración propia

\begin{tabular}{|c|c|c|c|c|c|c|c|}
\hline \multirow{2}{*}{\multicolumn{2}{|c|}{$\begin{array}{l}\text { Componente del } \\
\text { electrodo }\end{array}$}} & \multicolumn{6}{|c|}{$\begin{array}{c}\text { Por ciento, en masa, del elemento químico en cada componente del } \\
\text { electrodo }\end{array}$} \\
\hline & & $\mathrm{Mn}$ & $\mathrm{Cr}$ & $\mathrm{C}$ & $\mathrm{Si}$ & $\mathrm{Ti}$ & $\mathrm{Fe}$ \\
\hline & Alambre & 0,48 & 0,11 & 0,11 & 0,4 & 0,002 & Balance \\
\hline \multirow{3}{*}{ 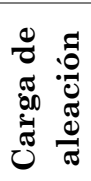 } & Ferrocromo & 0,00 & 75,00 & 0,10 & 2,00 & 0,00 & Balance \\
\hline & $\begin{array}{l}\text { Ferrocromo- } \\
\text { manganeso }\end{array}$ & 59,02 & 19,45 & 0,11 & 2,17 & 0,00 & Balance \\
\hline & Grafito & 0,00 & 0,00 & 99,50 & 0,00 & 0,00 & Otros \\
\hline
\end{tabular}

Tabla 2. Composición química del revestimiento del electrodo E6012. Fuente: elaboración propia.

\begin{tabular}{cccccccc}
\hline Composición en \% & $\mathrm{SiO}_{2}$ & $\mathbf{A l}_{\mathbf{2}} \boldsymbol{O}_{3}$ & $\mathbf{T i O}_{2}$ & $\mathbf{N a}$ & $\mathbf{C a}$ & $\boldsymbol{F e M}$ & $\boldsymbol{C}_{6} \mathrm{H}_{10}$ \\
\hline $\mathbf{E 6 0 1 2}$ & 16 & 6 & 52 & 2,5 & 2,5 & 14 & 7 \\
\hline
\end{tabular}



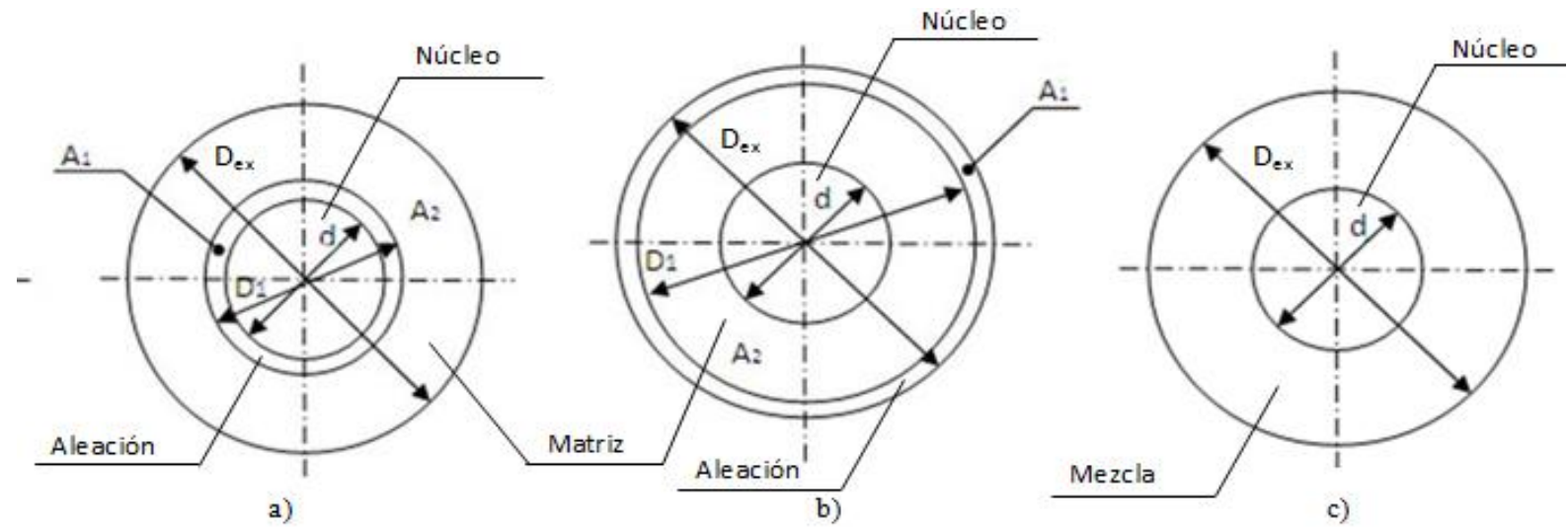

Fig. 1. Esquemas geométricos de la conformación de los revestimientos en los electrodos a) doblemente revestido (la carga de aleación interna) b) doblemente revestido (la carga de aleación externa) c) simplemente revestido

Fuente: elaboración propia.

Para la investigación, se estableció como estrategia comparativa la formulación de tres prototipos de electrodos, cuyas diferencias no estuvieran en su composición, sino en la posición que ocupan la carga de aleación y la matriz en la estructura del revestimiento del electrodo Fig. 1.

Como resultado se obtuvieron las dimensiones de los diferentes revestimientos para cada variante de electrodo desarrollado, Tabla 3.

\subsection{Métodos}

La planificación experimental se estableció para satisfacer el objetivo del trabajo relativo a la evaluación del efecto de la posición que ocupa la carga de aleación (C-Cr-Mn) en la estructura del revestimiento de un electrodo para el recargue. Se tomó la posición de la carga de aleación en la estructura del revestimiento como factor independiente.

Por las características del experimento, fue conveniente el uso de un diseño completamente al azar (DCA) [19], [20].

El juego de hipótesis a contrastar al aplicar la prueba ANOVA a los datos, según el DCA, fue [19]:

H1: Algún par de medias de los tratamientos difieren $(\mu \mathrm{i} \neq \mu \mathrm{j}$ para algún $\mathrm{i}$ $\neq \mathrm{j} ; \mathrm{i}=1,2,3$ )

Como herramienta para procesar esta parte del estudio se empleó el software Statgraphics Centurion XV [21].

Ho: Las medias de los tratamientos son iguales $\left(\mu_{1}=\mu_{2}=\mu_{3}\right)$

Criterio de expertos

El método de criterio de expertos aplicado permite ordenar factores que emitan los expertos a partir del juicio.

Tabla 3. Dimensiones de los electrodos. Fuente: elaboración propia.

\begin{tabular}{lcccc}
\hline Tipo de electrodo & Núcleo (d) & $\begin{array}{c}\text { Diámetros (mm) } \\
\text { Revestimiento } \\
\text { interior (Din) }\end{array}$ & $\begin{array}{c}\text { Revestimiento } \\
\text { exterior (Dex) }\end{array}$ & $\begin{array}{c}\text { Pesos } \\
(\mathbf{( g )})\end{array}$ \\
Con carga de aleación interna & 3,0 & 4,5 & 6,5 & 22,764 \\
Con carga de aleación externa & 3,0 & 5,8 & 6,5 & 22,766 \\
Con carga de aleación homogénea & 3,0 & - & 6,5 & 22,763 \\
\hline
\end{tabular}


Este método cuenta con los siguientes pasos: a) Selección del número de expertos; b) elaboración de la matriz de rangos con los criterios de los expertos a partir de la recolección de la información obtenida del juicio de cada experto; c) determinar el nivel de concordancia y realizar la validación del criterio de expertos [22].

El número óptimo de expertos se calculó en la expresión (1) a partir de la ecuación empleada por Duffus [23] en (1).

$$
\mathrm{n}=\frac{\mathrm{N}\left(\frac{\mathrm{i}^{2}}{\mathrm{k}}\right)+\mathrm{N}\left(\mathrm{p}-\mathrm{p}^{2}\right)}{\mathrm{N}\left(\frac{\mathrm{i}^{2}}{\mathrm{k}}\right)+\mathrm{p}-\mathrm{p}^{2}}
$$

Donde, $\mathrm{n}$ es el número óptimo de expertos a seleccionar; i, nivel de precisión que se estima de 0,$2 ; \mathrm{p}$, proporción del error que es de 0,$10 ; \mathrm{k}$, para un $95 \%$ de fiabilidad su valor es 3,84 y se identificó una población de expertos, $\mathrm{N}=4$.

Para procesar los datos recopilados se empleó el software profesional para procesamiento estadístico SPSS vs-23. Los criterios de estadística descriptiva se emplearon para conocer la evaluación del procedimiento respecto a cada uno de los principios que debe cumplir (el coeficiente de concordancia W de Kendall). Se calculó, además, el Índice de Validez de Contenido (IVC). Para estimar la fiabilidad del instrumento se empleó el estadígrafo alfa de Cronbach, el cual calcula la consistencia interna entre los parámetros que evalúan los expertos.

El criterio emitido por los expertos también se sometió al tratamiento estadístico anterior, y luego se obtuvieron las ecuaciones de regresión, que permiten obtener el peso de cada parámetro en estudio durante la definición de la mejor variante en el estudio de optimización.

\section{RESULTADOS Y DISCUSIÓN}

Se empleó un estudio de optimización por la complejidad de la valoración simultánea de todas las propiedades estudiadas y para identificar cuál de los electrodos podría tener un mejor desempeño.

\subsection{Identificación de los factores independientes a considerar}

Se identificó que los ensayos asociados al control del proceso de fabricación, como la resistencia mecánica y la excentricidad, satisfacen las normas empleadas, no aportando elementos de diferenciación entre las variantes de electrodos estudiados. Sin embargo, la higroscopicidad muestra diferencias, que pueden ser compensadas al aplicar los procedimientos estándares empleados durante la producción, conservación y uso de electrodos convencionales. En sentido general, si bien los ensayos valorados anteriormente sirven para evidenciar la calidad y reproducibilidad del comportamiento de los electrodos estudiados, los ensayos asociados al control de la calidad del proceso de fabricación no ofrecen parámetros robustos para ser considerados en una optimización.

Como parte de la evaluación del comportamiento operativo, se reconocieron como fundamentales los ensayos de estabilidad del arco y los relacionados con la determinación de los parámetros de consumo de los electrodos.

Se consideró la estabilidad del arco como uno de los indicadores operacionales principales del proceso de soldadura. Por otra parte, la estabilidad eléctrica del arco de soldadura constituye uno de los criterios de aceptabilidad o de calidad más reconocidos por los soldadores al emplear los electrodos. Además, existe el consenso de que un proceso de soldadura estable es el capaz de garantizar constancia en las dimensiones geométricas del cordón en toda su extensión [24]. Uno de los indicadores más robustos empleados para evaluar la estabilidad del arco en corriente alterna lo constituye la variación de la tasa 
media de incremento de la conductividad del espacio de arco $\left(\mathrm{B}^{+}\right)$, que contiene variables energéticas esenciales del proceso. Además, ha sido validado $\mathrm{y}$ aplicado en múltiples estudios [25].

Por otra parte, la norma para la determinación de los parámetros de fusión y deposición [6] refiere en su alcance su suficiencia de uso práctico para medir con precisión las variaciones en el comportamiento de cada electrodo individualmente en cuanto a eficiencia, recuperación de material y coeficiente de deposición. Este último parámetro representa la masa de metal depositado por amperes y minutos de un electrodo dado, bajo las condiciones establecidas.

Este indicador no depende de otros parámetros geométricos del electrodo (largo total, longitud de colilla, etc.), lo cual lo hace más robusto para la comparación del comportamiento entre electrodos.

Para la evaluación de la calidad del metal depositado se realizaron ensayos para la determinación de la composición química, la caracterización microestructural, la medición de la dureza y la resistencia al desgaste. Dentro de ellos, la resistencia al desgaste en los depósitos constituye un ensayo definitivo de calidad del depósito de un electrodo destinado a disminuir el desgaste abrasivo [26]. Para la evaluación de la resistencia al desgaste se toman los datos resultantes del ensayo de "abrasión con espiga", siendo el parámetro seleccionado la pérdida de masa, la cual resulta de la diferencia obtenida por medición gravimétrica de la masa inicial y final de la espiga ensayada, tomada durante un tiempo de $10 \mathrm{~min}$.

La norma [27] considera como válido para la comparación de los resultados para ensayos realizados con el mismo tipo $y$ grado de abrasivo.
Se consideran valores óptimos al evaluar el coeficiente de deposición $11,00 \cdot 10^{-3}$ y $14,00 \cdot 10^{-3} \mathrm{~kg} \cdot(\mathrm{A} \cdot \mathrm{h})^{-1}$ y en el caso de la conductividad del arco $(\mathrm{B}+)$ valores por encima de $110(\Omega \cdot \mathrm{s})^{-1}$ [27]. En el caso de la pérdida de masa, depende de las condiciones específicas del ensayo.

\subsection{Aplicación del criterio de expertos}

El número de expertos necesario en la expresión (1) fue de 3,04, por lo que se asumió una cantidad de expertos de tres.

Para seleccionar los integrantes más idóneos de la población considerada, se aplicó una encuesta estructurada para determinar el nivel de competencia de cada experto. Seguidamente se sometió a criterio de los expertos el procedimiento a emplear, para evaluar si posee validez de contenido (IVC); es decir, que posee carácter sistémico, consistencia lógica, equilibrio entre cada fase, flexibilidad y pertinencia [23]. El índice de validez de contenido (IVC) que se obtuvo es de 0,83 ; este valor es suficiente para valorar positivo el criterio del grupo respecto al ítem de evaluación.

El nivel de concordancia de los jueces (W Kendall) que se obtuvo es de 0,667 y la fiabilidad por el estadígrafo de alfa de Cronbach es 0,831 , lo cual ratifica la herramienta como fiable.

\subsection{Resultados de la aplicación del criterio de expertos a la selección de la mejor variante}

A los expertos se les entregó una encuesta con los resultados aleatorizados de todos los experimentos y sus respectivas réplicas, cuyos resultados se conjugaron posteriormente con la codificación inicial en Tabla 4. 
Metodología para la selección de la mejor variante entre electrodos de doble revestimiento para recargue

Tabla 4. Resultados de la aplicación de la encuesta. Fuente: elaboración propia.

\begin{tabular}{|c|c|c|c|c|c|c|c|}
\hline \multirow{2}{*}{$\begin{array}{l}\text { Número de las } \\
\text { corridas }\end{array}$} & \multirow{2}{*}{$\begin{array}{c}\text { Coeficiente de } \\
\text { deposición } \\
\left(\mathrm{g} \cdot \mathrm{A}^{-1} \cdot \min ^{-1}\right) \\
\mathrm{X}_{1}\end{array}$} & \multirow{2}{*}{$\begin{array}{c}\text { Pérdida de } \\
\text { masa } \\
(\mathrm{g}) \\
\mathrm{X}_{2}\end{array}$} & \multirow{2}{*}{$\begin{array}{c}\text { Conductividad } \\
\text { del arco }\left(\mathrm{B}^{+}\right) \\
\left(\Omega^{-1} \cdot \mathrm{s}^{-1}\right) \\
\mathrm{X}_{3}\end{array}$} & \multicolumn{3}{|c|}{$\begin{array}{c}\text { Criterios de } \\
\text { expertos }\end{array}$} & \multirow{2}{*}{$\begin{array}{c}\text { Codificación } \\
\text { Inicial }\end{array}$} \\
\hline & & & & $\mathrm{A}$ & B & $\mathrm{C}$ & \\
\hline 1 & 0,13 & 0,018 & 385,12 & 9 & 8 & 7 & Internas \\
\hline 2 & 0,14 & 0,015 & 334,82 & 4 & 5 & 6 & Homogéneas \\
\hline 3 & 0,16 & 0,008 & 268,05 & 2 & 2 & 2 & Externas \\
\hline 4 & 0,13 & 0,018 & 386,42 & 8 & 9 & 8 & Internas \\
\hline 5 & 0,14 & 0,015 & 290,93 & 6 & 4 & 4 & Homogéneas \\
\hline 6 & 0,16 & 0,007 & 220,23 & 3 & 3 & 3 & Externas \\
\hline 7 & 0,16 & 0,008 & 270,02 & 1 & 1 & 1 & Externas \\
\hline 8 & 0,14 & 0,016 & 305,35 & 5 & 6 & 5 & Homogéneas \\
\hline 9 & 0,13 & 0,018 & 344,26 & 7 & 7 & 9 & Internas \\
\hline
\end{tabular}

Atendiendo al ordenamiento realizado por los expertos (Tabla 4), según los criterios anteriormente establecidos, se pueden conformar tres grupos de resultados: grupo $\mathrm{A}$, con los números de las corridas 7, 3 y 6; grupo $\mathrm{B}$, con los números de las corridas 8,5 y 2 ; y grupo $\mathrm{C}$, con los números de las corridas 9,4 y 1 . Cada grupo puede asociarse con la denominación externa, homogénea e interna, respectivamente (Tabla 4).

Los expertos reconocen las características producidas por la aplicación de la carga de aleación en la parte externa del electrodo como las de mejor desempeño, continuando en su ordenamiento, de forma descendente, con los electrodos que poseen la carga distribuida homogéneamente, $y$, por último, los que contiene la carga de aleación ubicada internamente.
Se obtuvo un nivel de concordancia de los jueces (W Kendall) para los juicios emitidos de 0,941, y la fiabilidad por el estadígrafo de alfa de Cronbach es 0,969, lo cual reconoce a la herramienta como muy fiable.

El ordenamiento realizado por los expertos se procesó para obtener ecuaciones de regresión en Tabla 5 que permiten explicar matemáticamente el peso de las consideraciones asignadas por los expertos a cada factor estimado sobre el conjunto de resultados evaluados.

De la Tabla 5 se puede apreciar en la pérdida de masa que el factor $\mathrm{X}_{2}$ es el que mayor significación obtuvo en la evaluación de los expertos, siguie ndo en orden descendente el coeficiente de deposición $\left(\mathrm{X}_{1}\right)$ y la conductividad del arco $\left(\mathrm{X}_{3}\right)$.

Tabla 5. Ecuación de regresión obtenida según el criterio de evaluación de cada experto Fuente: elaboración propia.

\begin{tabular}{|c|c|c|c|}
\hline Experto & Ecuación de regresión & $\mathrm{R} 2$ & $\begin{array}{c}\text { No. } \\
\text { ecuación }\end{array}$ \\
\hline 1 & $y=73,027 X_{1}-277,978 X_{2}-0,005 X_{3}$ & 0,966 & $(2)$ \\
\hline 2 & $y=82,745 X_{1}-153,506 X_{2}-0,015 X_{3}$ & 0,973 & $(3)$ \\
\hline 3 & $y=76,415 X_{1}-241,581 X_{2}-0,008 X_{3}$ & 0,971 & (4) \\
\hline Combinación & $y=77,396 X_{1}-224,355 X_{2}-0,009 X_{3}$ & 0,966 & (5) \\
\hline
\end{tabular}


Este resultado muestra, tanto en la evaluación individual (Ecuaciones de la 2 a la 4) como en la integrada (Ecuación 5), la importancia que los expertos le confieren al factor dependiente "resistencia al desgaste", contraria a la que le dan a la estabilidad del arco. Esto se debe a que el valor de $\mathrm{B}^{+}$está dentro del rango de una conductividad eléctrica aceptable en las tres variantes.

El análisis (Tabla 5) muestra que la variante con mejores prestaciones para el proceso de recargue es la que posee la carga de aleación ubicada externamente en la estructura del revestimiento de los electrodos evaluados. Además, esta variante constituye un nuevo prototipo de electrodo doblemente revestido, caracterizado por presentar un revestimiento interno ordinario, empleada para electrodos de soldadura (E6012), y otra externa, compuesta por una carga de aleación (C-Cr-Mn), cuya aplicación es la aportación de recargues resistentes al desgaste abrasivo.

\section{CONCLUSIONES}

Los expertos reconocen que los parámetros más representativos para someter al criterio de los expertos son los resultados de la variación de la tasa media de incremento de la conductividad del espacio de arco (la estabilidad del arco), del coeficiente de deposición (parámetros de fusión y deposición) y del ensayo de abrasión con espiga (resistencia al desgaste).

Se selecciona unánimemente como la mejor variante de electrodo doblemente recubierto la que posee la carga de aleación externa, seguida de la variante con carga de aleación homogéneamente distribuida y, por último, la que posee la carga de aleación interna, a partir de una relevancia asignada a las variables de pérdida de masa (224), coeficiente de deposición (77) y estabilidad del arco $(0,01)$.
Los resultados del análisis realizado para la selección de la mejor variante sugieren que la resistencia al desgaste, evaluada a través de la pérdida de masa, es el criterio de mayor relevancia a considerar en la metodología para la selección de electrodos destinados al recargue de piezas.

\section{REFERENCIAS}

[1] E. S. Surian, "Consumibles para soldadura por arco eléctrico: algunos desarrollos recientes", Soldag. insp., Vol. 18, no. 1, pp. 77-89, Jan. 2013.

https://doi.org/10.1590/S010492242013000100011

[2] N. M. R de Rissone; J. P. Farias; I. de Souza Bott T; E. S. Surian, "ANSI/AWS A5.1-91 E6013 Rutile Electrodes: The Effect of Calcite All-weld-metal mechanical properties and microstructure, arc stability, and operational characteristics were investigated." Welding Journal, Supplement, pp. 112-124. Jul. 2002 URL

[3] A. Cruz Crespo; M. Rodriguez Pérez; A. Scotti, "Estudio comparativo de los parámetros de consumo y geometría de los depósitos de electrodos tubulares revestidos para recargue," en $8^{\circ}$ Congreso iberoamericano de ingenieria mecanica, Cusco, 2007, pp. 83- 93. URL

[4] E. Rabinowicz, Friction and Wear of Materials. 2nd ed.. John Wiley \& Sons. 2018. URL

[5] J. A. Pérez Cepeda, "Influencia de la microestructura en el comportamiento al desgaste abrasivo evaluado bajo norma astm $\mathrm{g}$ 65 de depósitos de soldadura antidesgaste aplicados sobre sustratos de acero de baja aleación y bajo carbono". (Tesis de Maestría), Facultad de Ingeniería, Departamento de Ingeniería Mecánica y Mecatrónica, Bogotá: Universidad Nacional de Colombia, 2011. URL

[6] ISO 2401-72. Covered Electrodes Determination of the Efficiency, Metal Recovery and Deposition Coefficient. 1994. 5p. URL

[7] R. Valdés Arencibia; A. Piratelli-Filho; A. CruzCrespo; E. Díaz Cedré., "Incertidumbre de la medición de masa en la determinación de los parámetros de consumo de electrodos de recargue", Soldagem \& Inspeção, vol. 18, no. 2. pp. 110-117. Jun. 2013.

http://dx.doi.org/10.1590/S010492242013000200004

[8] E. Huanca Cayo; S.C. Absi Alfaro, "Welding stability assessment in the GMAW-S process based on fuzzy logic by acoustic sensing from 
Metodología para la selección de la mejor variante entre electrodos de doble revestimiento para recargue

arc emissions", Journal of achivements in material and manufacturing engineering, vol. 55, no. 1, pp. 18-25, Nov. 2012. URL

[9] Yu.N. Lankin, "Indicators of stability of the GMAW process". The Paton Welding J, Vol. 1, pp. 6-13. 2011. URL

[10] A. García Rodríguez, "Energía de ionización simple en la soldadura con electrodo revestido". Soldag. Insp. vol. 18, no. 1 pp. 072-076. Jan. 2013.

https://doi.org/10.1590/S010492242013000100010

[11] M. T. Assunção; A. Queiroz-Bracarense, "Evaluation of the effect of the water in the contact tip on arc stability and weld bead geometry in underwater wet FCAW". Soldag. Insp., vol. 22, no. 4, pp. 401-412. Oct. 2017. https://doi.org/10.1590/0104-9224/si2204.11

[12] M.Suban; J.Tušek, "Dependence of melting rate in MIG/MAG welding on the type of shielding gas used", Journal of Materials Processing Technology, vol 119, no. 1-3, pp. 185-192. Dec. 2001.

https://doi.org/10.1016/S0924-0136(01)00940-2

[13] A. García-Rodríguez, et al., Comportamiento del tiempo de duración, la frecuencia de los cortocircuitos y la conductividad eléctrica durante el reencendido del arco en la soldadura SMAW (AC) con electrodos E6013. Soldagem Insp. São Paulo, Vol. 14, No. 1, pp. 66-73, Jan. 2009. URL

[14] V. Ponomarev; O. Al-Erhayem; R. L. Apps; B. Lindberg, Arc Welding Process Statistical Analysis: Methodical Approaches, Analysis Conceptions, Experiences. Manual-guide, 1997.

[15] Z. Vivas Riveros, "Estudio de la resistencia al desgaste de películas delgadas de Ti”. (Trabajo de grado), Facultad de Ingenieria, Departamento de Energética y Mecánica. Universidad Autonoma de Occidente. Santiago de Cali. 2006. URL

[16] E. Palacio-Caballero, "Diseño y fabricación de una máquina de ensayo de desgaste abrasivo del tipo "pin sobre disco" según la norma ASTM G99-04". (Trabajo de grado), Facultad de Ingeniería Mecánica, Universidad Central "Marta Abreu" de las Villas. 2014. URL

[17] C. A. Nobre Cavalcanti, "Comparação entre o comportamento de eletrodos revestidos utilizando dispositivos de soldagem por gravidade e com controle automático do comprimento do arco elétrico" (Trabajo de grado), Facultad de Ingeniería Mecánica, Universidade Federal do Rio Grande do Soul, Porto Alegre, 2011. URL

[18] F. A. Díaz-Rosell, "Diseño Estadístico de Experimentos". (Trabajo de grado), Facultad Matemática, Física y Computación, Universidad Central "Marta Abreu" de Las Villas: Santa Clara. 2006. URL
[19] StatPoint, Inc- Diseño de Experimentos Diseños de un Solo Factor Categórico, STATGRAPHICSC 2006. por StatPoint, Inc. $\underline{\mathrm{URL}}$

[20] Statpoint Technologies, Centurion XVII User Manual, 2006. URL

[21] Martín, W.F., et al., Metodología de la investigación científica. Editorial Universidad de Cienfuegos. 2006. Cienfuegos: Cuba. pp. 137. Consultado: 03/10/2016. URL

[22] D. Duffus-Miranda; A. Cuellar-Ramallo; Z. Escobar-Díaz, "Estudio técnico para la evaluación de proyectos en la fase de pre inversión en Cuba", Revista Observatorio de la Economía Latinoamericana, pp. 1- 16, May. 2018. URL

[23] I. K. Pokhodnya, Metallurgy of Arc Welding: Arc processes and electrode melting. Riecansky Science Publishing CO. London .1995.

[24] J. P. Farias; A. M. Quites; E. S. Surian, "The Effect of Magnesium Content on the Arc Stability of SMAW E7016-C2L/8016-C2 Covered Electrodes Magnesium additions to the coating improved the charge and metal transfer during welding with alternating current". Welding Journal. Welding Research Suplement. 199776 (6) 245-250. URL

[25] A. Gualco; H. G. Svoboda; E. S. Suriana; L. A. de Vedia, "Effect of welding procedure on wear behaviour of a modified martensitic tool steel hardfacing deposit". Materials and Design Vol. 31, no. 9 pp. 4165-4173. https://doi.org/10.1016/j.matdes.2010.04.026

[26] Active Standard ASTM G132, Standard test method for pin abrasion testing.. International, West Conshohocken, PA, 2018. URL

[27] V D Makarenko; C P Shatilo, "Stability of arcing in welding with calcium fluoride electrodes," Welding International, vol. 4, no. 12 , pp. 988-989. 1990. https://doi.org/10.1080/09507119009453038

\section{CONTRIBUTIONS}

1 Conceptualización, revisión, validación, análisis formal del trabajo, recursos, realización de los ensayos, escritura, adquisición de datos, conformación del marco teórico, análisis estadístico.

(D) 2 Conceptualización, revisión, supervisión, realización de los ensayos.

(i) 3 Optimización, procesamiento matemático, ecuaciones de regresión. 